\title{
Failure of density-matrix minimization methods for linear-scaling density-functional theory using the Kohn penalty-functional
}

\author{
P. D. Haynes and M. C. Payne \\ Theory of Condensed Matter, Cavendish Laboratory, Madingley Road, Cambridge \\ CB3 OHE, United Kingdom
}

\begin{abstract}
We examine the recently-proposed scheme [W. Kohn, Phys. Rev. Lett. 76, 3168 (1996)] for performing linear-scaling calculations within density-functional theory by direct minimization with respect to the single-particle density-matrix using a penalty-functional to exactly enforce the idempotency constraint. We show that such methods are incompatible with standard minimization algorithms (using conjugate gradients as an example) and demonstrate that this is a direct result of the nonanalytic form of penalty-functional which must be chosen to obtain a variational

principle for the total energy.
\end{abstract}

PACS numbers: 71.15.Mb

The traditional formulation of density-functional theory [1] (DFT) in terms of a set of extended single-particle wave functions has led to the development of schemes for performing total-energy calculations which require a computational effort which scales as the cube of the system-size (i.e. the number of atoms, electrons or volume of the system). This scaling results from the cost of diagonalizing the Hamiltonian or orthogonalizing the wave functions. Methods based upon the single-particle density-matrix (DM), which is free from orthogonality constraints and short-ranged in real-space, offer the prospect of electronic structure calculations at a cost which scales only linearly with system-size. We investigate one scheme that has been proposed for achieving this goal, which uses a penalty-functional to impose the idempotency constraint on the DM. We show that the form of penalty-functional which must be chosen to obtain a variational principle for the total energy precludes the use of efficient minimization algorithms commonly used in electronic structure calculations. We apply the method to crystalline silicon and show that the desired minimum cannot be found and therefore that the variational principle cannot be used in practice. 
In terms of a set of orthonormal orbitals $\left\{\varphi_{i}\right\}$ and occupation numbers $\left\{f_{i}\right\}$, the DM $\rho$ is written as

$$
\rho\left(\mathbf{r}, \mathbf{r}^{\prime}\right)=\sum_{i} f_{i} \varphi_{i}(\mathbf{r}) \varphi_{i}^{*}\left(\mathbf{r}^{\prime}\right)
$$

For the ground-state DM, the $\left\{\varphi_{i}\right\}$ are eigenstates of the self-consistent KohnSham Hamiltonian with eigenvalues $\left\{\varepsilon_{i}\right\}$ and occupation numbers $\left\{f_{i}\right\}$ equal to unity or zero for states below or above the chemical potential $\mu$ respectively. The DM must be Hermitian and normalized (to correspond to a system of $N_{\mathrm{e}}$ electrons):

$$
N[\rho]=2 \int \mathrm{d} \mathbf{r} \rho(\mathbf{r}, \mathbf{r})=2 \sum_{i} f_{i}=N_{\mathrm{e}}
$$

where the factor of two arises from spin degeneracy. In addition, the groundstate DM must be idempotent:

$$
\rho^{2}\left(\mathbf{r}, \mathbf{r}^{\prime}\right)=\int \mathrm{d} \mathbf{r}^{\prime \prime} \rho\left(\mathbf{r}, \mathbf{r}^{\prime \prime}\right) \rho\left(\mathbf{r}^{\prime \prime}, \mathbf{r}^{\prime}\right)=\rho\left(\mathbf{r}, \mathbf{r}^{\prime}\right)
$$

The energy functional $E[\rho]$ is defined by

$$
E[\rho]=-\int \mathrm{d} \mathbf{r}^{\prime}\left[\nabla_{\mathbf{r}}^{2} \rho\left(\mathbf{r}, \mathbf{r}^{\prime}\right)\right]_{\mathbf{r}=\mathbf{r}^{\prime}}+E_{\mathrm{Hxc}}[n]+\int \mathrm{d} \mathbf{r} V_{\mathrm{ext}}(\mathbf{r}) n(\mathbf{r}),
$$

where $E_{\mathrm{Hxc}}[n]$ is the sum of the Hartree and exchange-correlation energies which depend only on the electronic density $n(\mathbf{r})=2 \rho(\mathbf{r}, \mathbf{r})$ and $V_{\text {ext }}$ is the external potential arising from the ions. The ground-state energy can be found by minimizing this functional with respect to all Hermitian, normalized and idempotent DMs. Without the idempotency constraint, the minimization is unstable with respect to unphysical DMs in which low-energy states are overoccupied (with more than two electrons each) and high-energy states are negatively occupied.

Exploiting the short-ranged behaviour of the DM, i.e. that $\rho\left(\mathbf{r}, \mathbf{r}^{\prime}\right) \rightarrow 0$ exponentially [2] as $\left|\mathbf{r}-\mathbf{r}^{\prime}\right| \rightarrow \infty$, by imposing some spatial cut-off $r_{\text {cut }}$ (such that $\rho\left(\mathbf{r}, \mathbf{r}^{\prime}\right)=0$ for $\left.\left|\mathbf{r}-\mathbf{r}^{\prime}\right|>r_{\text {cut }}\right)$ results in a linear-scaling method. The most significant hurdle to overcome is the imposition of the idempotency constraint. This can be achieved implicitly using a purifying transformation [3] which has been implemented in several tight-binding and DFT schemes [4].

An alternative approach to imposing the idempotency constraint has been proposed by Kohn [5], who suggested minimizing the functional $\tilde{Q}$ defined by

$$
\tilde{Q}[\rho ; \mu, \alpha]=E_{\mathrm{NI}}\left[\rho^{2}\right]-\mu N\left[\rho^{2}\right]+\alpha P[\rho]
$$


where $E_{\mathrm{NI}}$ is the total energy of the non-interacting Kohn-Sham system, $N$ is defined in (2), and the penalty-functional $P$ is

$$
P[\rho]=\left\{\int \mathrm{d} \mathbf{r}\left[\rho^{2}(1-\rho)^{2}\right]_{\mathbf{r}^{\prime}=\mathbf{r}}\right\}^{\frac{1}{2}}=\left[\sum_{i} f_{i}^{2}\left(1-f_{i}\right)^{2}\right]^{\frac{1}{2}} .
$$

Kohn derived a variational principle for the functional $\tilde{Q}$ which states that for values of $\alpha$ larger than some critical value, the minimum value of $\tilde{Q}$ is an upper bound to the ground-state grand potential of the system.

Rather than minimizing the non-interacting energy, as proposed by Kohn, we can instead minimize the interacting energy self-consistently. Using the square of the DM to calculate $E_{\mathrm{NI}}$ in (5) has the advantage that it guarantees that the charge density is positive-definite. However, in order to simplify the analysis here we consider the functional $Q$ defined by

$$
Q[\rho ; \mu, \alpha]=E[\rho]-\mu N[\rho]+\alpha P[\rho]
$$

where the interacting energy $E$ is defined by (4), $N$ by (2) and $P$ by (6). Using Janak's theorem [6] the derivative of $Q$ with respect to occupation numbers is

$$
\frac{\partial Q[\rho ; \mu, \alpha]}{\partial f_{i}}=2\left(\varepsilon_{i}-\mu\right)+\frac{\alpha}{P[\rho]} f_{i}\left(1-f_{i}\right)\left(1-2 f_{i}\right) .
$$

For the case of idempotent DMs, for which $P=0$, we obtain the special cases

$$
\left.\frac{\partial Q[\rho ; \mu, \alpha]}{\partial f_{i}}\right|_{f_{i}=\left\{0^{ \pm}, 1^{ \pm}\right\}}=2\left(\varepsilon_{i}-\mu\right) \pm \alpha,
$$

since, in this case, all variations away from the idempotent ground-state DM cause $Q$ to increase. We note that the first derivative of the functional is undefined for idempotent DMs. The ground-state idempotent DM will thus minimize $Q$ if $\alpha$ exceeds some critical value $\alpha_{\mathrm{c}}$ for which a lower bound is

$$
\alpha_{\mathrm{c}}>2 \max _{i}\left|\varepsilon_{i}-\mu\right|
$$

This is illustrated schematically in Fig. 1 for the case of a single occupation number corresponding to a state above the chemical potential. For $\alpha>\alpha_{\mathrm{c}}$ the minimum value of $Q$ is obtained when $f_{i}=0$. This outlines the variational principle established by Kohn, but we note that the functional $Q$ is minimal only in the sense that it takes its minimum value at the ground-state, but 

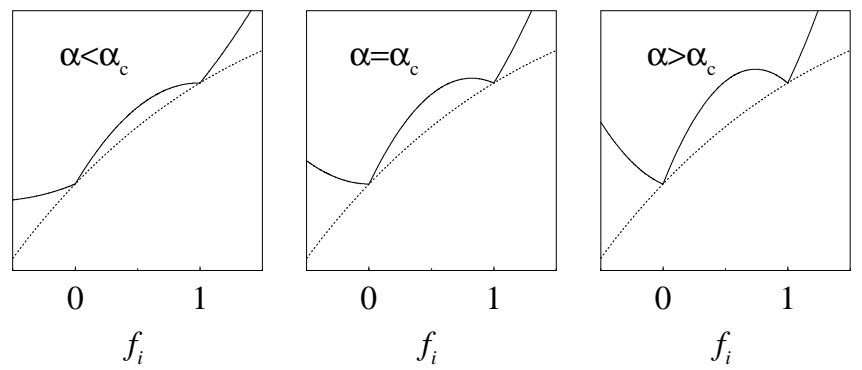

Fig. 1. Schematic illustration of the variational principle: behaviour of the grand potential (dotted) and total functional (full) for representative values of $\alpha$ when the occupation number of a single state above the chemical potential is varied.

not in the sense that its derivatives vanish at the ground-state, since they are undefined at that point.

The penalty-functional $P$ has a branch point at its minimum, due to the square-root form employed (6). However, this square-root is crucial to establishing the variational principle. In Fig. 2, the effect of using an analytic penalty-functional (the square of $P$ ) is plotted and it is clear that the minimum now occurs for $f_{i}<0$ for all values of $\alpha$. The total energy calculated in this case will no longer be a variational upper bound to the ground-state energy. We have recently introduced a method for obtaining accurate estimates of the true ground-state energy from such nearly-idempotent DMs, and details can be found elsewhere [7]. Nevertheless, the non-analytic form of the penalty-functional (6) must be employed if we wish to obtain a variational principle for the energy.

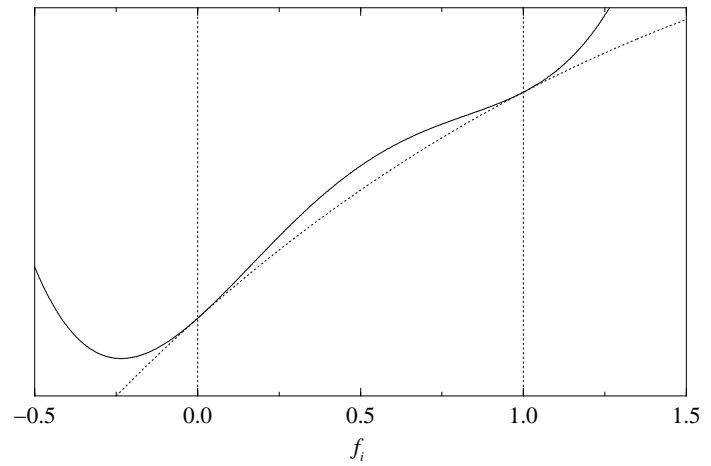

Fig. 2. Schematic illustration of the lack of a variational principle when an analytic penalty-functional is used. For the case of unoccupied bands, as shown here, the minimum occurs for $f_{i}<0$.

Several schemes exist for directly minimizing functions of many variables. The simplest of these is the method of steepest descents in which the gradient of the function is used as a search direction in the multidimensional space. The minimum value of the function along this direction is found and the process iterated to convergence. In Fig. 3a, the results of applying this method to an exactly quadratic function $f(x, y)=x^{2}+10 y^{2}$ are plotted, starting from 
the point $(5,1)$. Successive search directions are always orthogonal, and the method is not guaranteed to find the minimum in a finite number of steps. The method is clearly inefficient, since all of the information from previous function and gradient evaluations is ignored when calculating new search directions. In contrast, the conjugate gradients method [8] uses this information to construct independent search directions. Each successive step effectively eliminates one dimension of the space to be searched, so that the minimum is found in a number of steps no greater than the dimensionality of the space. The results for this method are plotted in Fig. 3b for the same quadratic function plotted in Fig. 3a. This method has been successfully implemented in traditional electronic structure calculations [9].
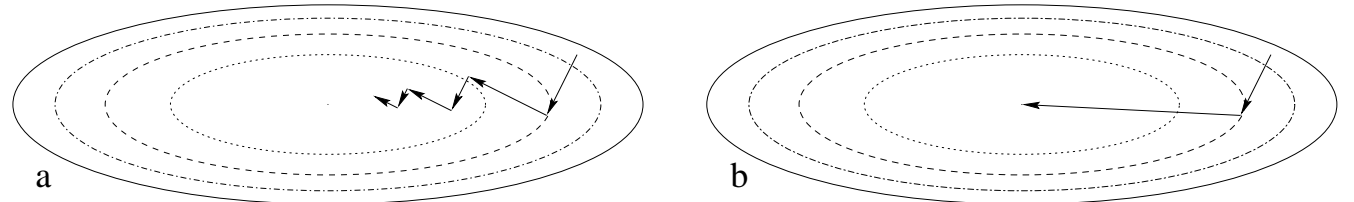

Fig. 3. Elliptic contours of a quadratic function showing search directions obtained by (a) the steepest descents method (directions are mutually orthogonal, and a large number of steps is required to find the minimum) and (b) the conjugate gradients method (only two steps required to find the minimum exactly).

However, the conjugate gradients method relies on the accuracy of a quadratic approximation of the function around the minimum. As observed, the functional $Q$ has a branch point at its minimum which arises from the squareroot that appears in the penalty-functional, and is therefore non-analytic at the minimum. No multidimensional Taylor expansion for the functional exists about the minimum, and so the local information (functional values and gradients at points) used to construct the conjugate directions gives a misleading picture of the global behaviour of the functional. In Fig. 4a the results of a steepest descents minimization of the function $g(x, y)=\sqrt{f(x, y)}=$ $\sqrt{x^{2}+10 y^{2}}$ is plotted, and exactly the same sequence of points is generated as in Fig. 3a. However, the results obtained using the conjugate gradients method, plotted in Fig. 4b, are now worse than for steepest descents.
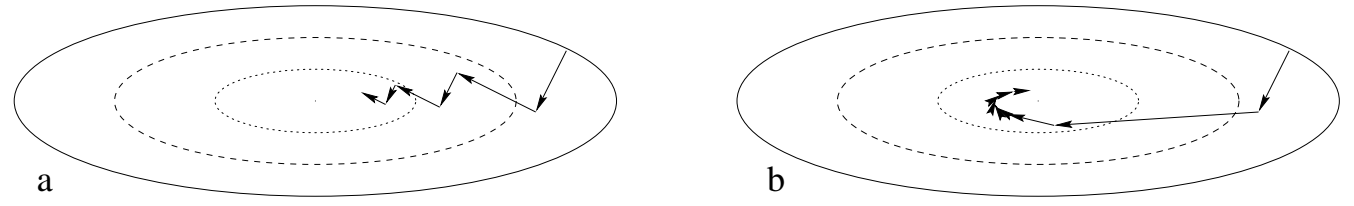

Fig. 4. Elliptic contours for a function with a branch point at the minimum (the square-root of the function plotted in Fig. 3) with search directions for (a) the steepest descents method (exactly the same sequence of points is generated as in Fig. 3a) and (b) the conjugate gradients method (now less efficient than steepest descents).

In Fig. 4, the exact line minimum is found in each case. In practice, however, the position of the line minimum is usually estimated, by making a parabolic 
interpolation of the functional along the search direction using the initial value and first derivative of the functional, and its value at a trial step. In this case, the line minimum estimate will also be wrong, further reducing the efficiency in both cases.

These problems are not confined to the conjugate gradients method alone, but apply equally to any method which attempts to use gradients to build up an estimate of the Hessian of the functional e.g. the Fletcher-Powell or BroydenFletcher-Goldfarb-Shanno algorithms [10]. The Car-Parrinello scheme [11] would also fail in this case since the derivative of the penalty-functional would appear in the equations of motion for the molecular-dynamics Lagrangian. Simulated annealing methods [12] such as the Metropolis algorithm [13] will successfully minimize functions of this kind, since they do not use the gradients, but the number of iterations required in this case would increase with system-size as the number of dimensions to be searched increased, thus spoiling the linear scaling of the method.

We can attempt to find a set of conjugate directions for the non-quadratic functions encountered here. For a function $f(\mathbf{x})$ with a quadratic minimum, a set of conjugate directions $\left\{\mathbf{d}_{k}\right\}$ are defined by

$$
\begin{aligned}
& \mathbf{d}_{1}=-\nabla f\left(\mathbf{x}_{0}\right), \\
& \mathbf{d}_{k+1}=-\nabla f\left(\mathbf{x}_{k}\right)+\gamma_{k} \mathbf{d}_{k} \quad(k>0)
\end{aligned}
$$

where $k$ labels the iteration and $\mathbf{x}_{k}$ is the position of the line minimum along the search direction $\mathbf{d}_{k}$. Several expressions for $\gamma_{k}$ exist, all of which are equivalent for exactly quadratic functions, and one of these is

$$
\gamma_{k}=\frac{\nabla f\left(\mathbf{x}_{k}\right) \cdot \nabla f\left(\mathbf{x}_{k}\right)}{\nabla f\left(\mathbf{x}_{k-1}\right) \cdot \nabla f\left(\mathbf{x}_{k-1}\right)} .
$$

For the function $g(\mathbf{x})=\sqrt{f(\mathbf{x})}$, the gradient $\nabla g(\mathbf{x})=[2 g(\mathbf{x})]^{-1} \nabla f(\mathbf{x})$ so that redefining $\gamma_{k}$ by

$$
\gamma_{k}=\frac{g\left(\mathbf{x}_{k}\right)}{g\left(\mathbf{x}_{k-1}\right)} \frac{\nabla g\left(\mathbf{x}_{k}\right) \cdot \nabla g\left(\mathbf{x}_{k}\right)}{\nabla g\left(\mathbf{x}_{k-1}\right) \cdot \nabla g\left(\mathbf{x}_{k-1}\right)}
$$

enables a set of conjugate directions to be obtained, which are parallel to the directions obtained for $f(\mathbf{x})$. For the example of the function plotted in Fig. 4, minimization using this new expression for $\gamma_{k}$ yields the same results as plotted in Fig. 3b. Such a scheme would therefore enable the efficient minimization of the penalty-functional $P$. However, for the functional $Q$ which consists of the sum of a functional with a quadratic minimum, $E-\mu N$, and 
the penalty-functional $P$ which has a branch point like $g(\mathbf{x})$ above at the minimum, neither expression for $\gamma_{k}$ is suitable, and we are unable to define a set of conjugate directions which can be used to simultaneously minimize both types of function.

When using the conjugate gradients method to minimize functions which are not exactly quadratic in form, it is common practice to reset the conjugate directions after a certain number of iterations by taking a steepest descent step. For functions of the same form as $Q$, the method becomes rapidly less efficient as the number of successive conjugate gradients steps is increased for the reasons discussed above. Indeed, for functions of many variables the method may completely fail to find the true minimum. Instead, the method appears to converge to a value which is not the minimum. This trend can be seen in Fig. 4b, in which the conjugate directions become orthogonal to the gradient. Therefore, the method fails not as a result of false minima, but because the minimization becomes so slow as to be indistinguishable from true convergence.

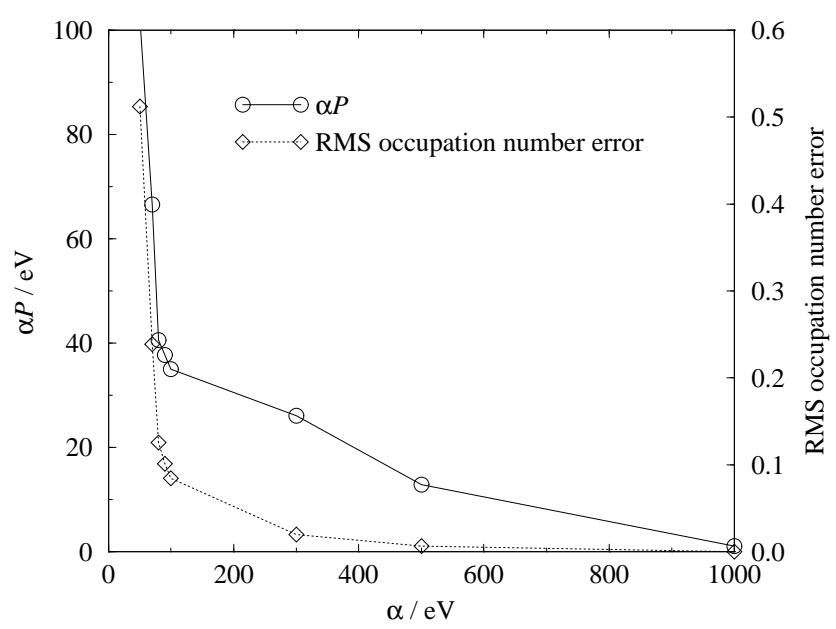

Fig. 5. Variation of the contribution of the penalty-functional to the minimized total functional and the RMS error in the occupation numbers with respect to $\alpha$ for crystalline silicon.

In order to see whether such behaviour appears in a genuine DFT calculation, we have implemented this scheme to perform total energy calculations, and have applied it to crystalline silicon. The density-matrix was expanded in separable form in terms of a sparse Hermitian matrix and a set of localized functions, as in other schemes [4], and the localized functions themselves were centred on the ions and expanded in terms of a localized spherical-wave basisset [14] using an energy cut-off of $200 \mathrm{eV}$ and angular momentum components up to $\ell=2$. No attempt was made to converge the calculation with respect to the density-matrix cut-off. The variational principle proved by Kohn states that for $\alpha>\alpha_{\mathrm{c}}, P=0$ at the minimum. In Fig. 5, we show the contribu- 
tion of the penalty-functional $\alpha P$ to the total minimized functional $Q$. As $\alpha$ is increased, this contribution does not vanish above some critical value (estimated from (10) and by Kohn's limit to be of the order of $50 \mathrm{eV}$ for silicon) but rather decreases slowly. Also plotted in Fig. 5 is the corresponding root-mean-square error in the occupation numbers, which also decreases as $\alpha$ increases. Thus the total energy $E$ will approach the true ground-state value, but no variational principle can be invoked, since this only holds for $P=0$ exactly. This failure to observe Kohn's result in practice is due to the inability of the minimization procedure to locate the true minimum of the functional, due to its non-analytic form.

In conclusion, we have implemented a method based upon the variational principle derived by Kohn [5] and demonstrated a fundamental difficulty in using this method in a computational scheme which is due to the non-analyticity of the required penalty-functional. Calculations on crystalline silicon confirm the trends observed from considering simple model functions. We have shown that the variational principle cannot be exploited in practice because the nature of the functional makes it unsuitable for use with current minimization techniques. Other schemes based upon the purifying transformation, or which use an analytic penalty-functional to approximately impose idempotency (and subsequently correct for the error introduced due to the lack of idempotency) will therefore be more efficient.

We acknowledge helpful discussions with E. Sandré. PDH was supported by an EPSRC studentship.

\section{References}

[1] Hohenberg P. and Kohn W., Phys. Rev. 136, 864 (1964); Kohn W. and Sham L. J., ibid. 140, 1133 (1965).

[2] Ismail-Beigi S. and Arias T. (unpublished).

[3] McWeeny R., Rev. Mod. Phys. 32, 335 (1960).

[4] Li X.-P., Nunes R. W. and Vanderbilt D., Phys. Rev. B 47, 10891 (1993); Daw M. S., ibid. 47, 10895 (1993); Hernández E., Gillan M. J. and Goringe C. M., ibid. 53, 7147 (1996); Daniels A. D., Millam J. M. and Scuseria G. E., J. Chem. Phys. 107, 425 (1997).

[5] Kohn W., Phys. Rev. Lett. 76, 3168 (1996).

[6] Janak J. F., Phys. Rev. B 18, 7165 (1978).

[7] Haynes P. D. and Payne M. C. (unpublished). 
[8] Gill P. E., Murray W. and Wright M. H., Practical Optimization (Academic, London, 1981).

[9] Štich I., Car R., Parrinello M. and Baroni S., Phys. Rev. B 39, 4997 (1989); Teter M. P., Payne M. C. and Allan D. C., ibid. 40, 12255 (1989); Gillan M. J., J. Phys.: Condens. Matter 1, 689 (1989).

[10] Press W. H., Teukolsky S. A., Vetterling W. T. and Flannery B. P., in Numerical Recipes (Cambridge University, England, 2nd Ed., 1994) pp. $418 \mathrm{ff}$.

[11] Car R. and Parrinello M., Phys. Rev. Lett. 55, 2471 (1985).

[12] Kirkpatrick S., Gelatt C. D. and Vecchi M. P., Science 220, 671 (1983).

[13] Metropolis N. et al., J. Chem. Phys. 21, 1087 (1953).

[14] Haynes P. D. and Payne M. C., Comp. Phys. Comm. 102, 17 (1997). 